\title{
Polar decomposition of the Mueller matrix: a polarimetric rule of thumb for square-profile surface structure recognition
}

\author{
J. M. Sanz, ${ }^{\star}$ J. M. Saiz, F. González, and F. Moreno \\ Departamento de Física Aplicada, University de Cantabria, Avda. Los Castros, s/n. 39005-Santander, Spain \\ ${ }^{*}$ Corresponding author: sanzjm@ unican.es
}

Received 15 February 2011; revised 3 May 2011; accepted 24 May 2011; posted 26 May 2011 (Doc. ID 142589); published 11 July 2011

\begin{abstract}
In this research, the polar decomposition (PD) method is applied to experimental Mueller matrices (MMs) measured on two-dimensional microstructured surfaces. Polarization information is expressed through a set of parameters of easier physical interpretation. It is shown that evaluating the first derivative of the retardation parameter, $\delta$, a clear indication of the presence of defects either built on or dug in the scattering flat surface (a silicon wafer in our case) can be obtained. Although the rule of thumb thus obtained is established through PD, it can be easily implemented on conventional surface polarimetry. These results constitute an example of the capabilities of the PD approach to MM analysis, and show a direct application in surface characterization. (C) 2011 Optical Society of America
\end{abstract}

OCIS codes: $\quad 120.2130,120.5410,120.5820,120.0120,290.0290,050.0050$.

\section{Introduction}

For a scattering system illuminated by a given incident wavelength, the Mueller matrix (MM) is a complete polarimetric result, in the sense that it contains all information about the scattering properties of a system, as far as intensity and polarization of the scattered radiation are concerned. The measurement of the MM is a way to characterize both the geometry and optical properties of a scattering system, a nonunique process and a part of the so-called inverse problem. There has been much work trying to exploit its capabilities, in the line of connecting MM properties to those, either optical or geometrical, of the scattering system [1-3]. For most cases, however, the elements of the $\bar{M} \bar{M}$ contains encrypted information, and are not easily related to the properties of the system, unless some kind of transformation is introduced. Pursuing this idea, MM decomposition, i.e., expressing it as a product-or sum-of several

0003-6935/11/213781-08\$15.00/0

(C) 2011 Optical Society of America matrices, has become popular in recent years. Polar decomposition (PD) [4], in particular forward PD [5], introduces three matrices in the following way:

$$
M=M_{\Delta} M_{R} M_{D},
$$

representing independent physical actions of the system on the polarimetric properties of the scattered light, respectively: $M_{\Delta}$ (depolarization), $M_{R}$ (retardation), and $M_{D}$ (diattenuation). If it is proved that a particular feature of the system is related to any of the parameters contained in such matrices, a more direct way of analysis is then opened. Since the PD is an MM algebraic transformation, all results obtained can be applied to conventional polarimetry through an adequate manipulation of the elements $m_{i j}$. The PD method has been used in medicine [6] to improve results in tissue exploration [7], and in other applications, like imaging polarimetry [8] or grating surfaces characterization [9]. Here, $\overline{\mathrm{P} D}$ is applied to a set of MMs experimentally obtained for a flat surface system, a silicon wafer, containing similar 
square-profiled structures that are either built on or dug in the surface (we shall refer to them as ribs or grooves, respectively). Au sputtering is used to introduce the metal character of the sample. The goal of this research is to distinguish between both cases, through PD analysis, in a systematic way for all the sizes and materials analyzed, and following a single check, or rule of thumb. Therefore, for a particular sample, there would be no need for individual analysis of patterns, or comparisons with numerical results, in order to know to which family of microstructures it belongs.

\section{Experimental Setup}

\section{A. Dual Rotating Compensator Polarimeter (DRCP)}

The DRCP developed in our labs consists of a $\mathrm{He}-\mathrm{Ne}$ laser, a polarization state generator (PSG), composed of a polarizer and a rotating quarter-wave plate, a sample holder, a polarization state analyzer (PSA), composed of a rotating quarter-wave plate and an analyzer, and a detector. A long focal-length lens is placed after the laser to control the spot size $(0.6 \mathrm{~mm}$ at the sample location) and beam divergence. Quarter-wave plates rotate synchronously with a speed ratio of $5: 2$ [10], and the joint transmittance describes a Fourier cycle [11], which is related to the polarizer azimuth and the quarter-wave plates azimuthal origin and true retardance. This Fourier cycle can be used to obtain the complete MM of a scattering (or either transmitting or reflecting) sample, located between the PSG and the PSA, by means of a numerical inverse Fourier transform. The sample is on a rotating stage in order to control the angle of incidence (normal incidence), and the PSA-detector couple is mounted on the arm of a rotor to select the scattering angle (from $\theta= \pm 90^{\circ}$ to $\theta= \pm 20^{\circ}$ ). Sample holder is on a tilt rotating platform with a $X Y Z$ nanopositioner in order to allow an accurate alignment [Fig. 1(a) shows a schematic vision of the PDRC]. The angular step between measurements is $1^{\circ}$ for a calculated resolution of $0.25^{\circ}$.

\section{B. Samples}

The samples analyzed in this work consist of a flat surface system containing square-profiled structures that are either built on or dug in the surface (ribs or grooves, respectively). These two-dimensional (2D) geometries were designed as a configuration with strong, but well-characterized, interference and diffraction, and with the presence of multiple scattering effects in a variable degree. The set of structures analyzed includes one or two ribs/grooves with a depth/ height $(h)$ of 1 or $2 \mu \mathrm{m}$, a width $(w)$ ranging from 1 to $4 \mu \mathrm{m}$, and with distance between centers $(d)$ ranging from 4 to $8 \mu \mathrm{m}$. The target is a Silicon wafer, structured by photolithographic techniques (microetching) and its composition is either $\mathrm{Si}$ or $\mathrm{Au}$ sputtered. It is divided in sections, each one containing a different sample. All these possibilities offer a complete set of configurations with varying depth sign, width, height, number, and optical properties. Figures 1(b) and 2 show, respectively, the scattering configuration and a scanning electron microscopy (SEM) image with a dimensional scheme corresponding to a two-rib sample.

\section{PD Method}

The experimental scattering matrices have been postprocessed with an algorithm that performs the PD. After testing the physical reliability of the matrices by the Cloude's coherency criterion [12], it was found that, in all the cases analyzed, the MMs obtained were reliable and the application of a PD algorithm is justified. Forward PD [5] is a kind of PD defined as a product of a depolarization matrix, $M_{\Delta}$, a retardation matrix, $M_{R}$, and a diattenuation matrix, $M_{D}$, as shown in Eq. (1). The first matrix, $M_{D}\left(t_{1}, t_{2}, \alpha, \beta\right)$ in order of operation, is the diattenuation matrix [4]. Parameters $\alpha$ and $\beta$ are the azimuth and ellipticity of the diattenuator principal axis, respectively, and $t_{i}$ is the transmittance through the $i$ principal axes, i.e., the intensity transmission for the diattenuator $i$ orthogonal polarization eigenstate. If the diattenuation matrix is normalized to the total transmittance for unpolarized light $m_{00}$, both transmittances along the diattenuator axes are related: $t=t_{1}=1-t_{2}$ [13]. Then,

$$
M_{D}\left(t_{1}, t_{2}, \alpha, \beta\right)=m_{00} \cdot M_{D}^{\prime}(t, \alpha, \beta) .
$$

The second matrix, $M_{R}$, contains a retardation parameter, $\delta$ (representing the ability of the system to introduce some phase retardation between both orthogonal components or retarder eigenstates), an azimuthal parameter, $\phi$ (that represents the eigenstates orientation origin), and the optical rotation, $\rho$ [7]. $M_{R}$ can be expressed as [14]
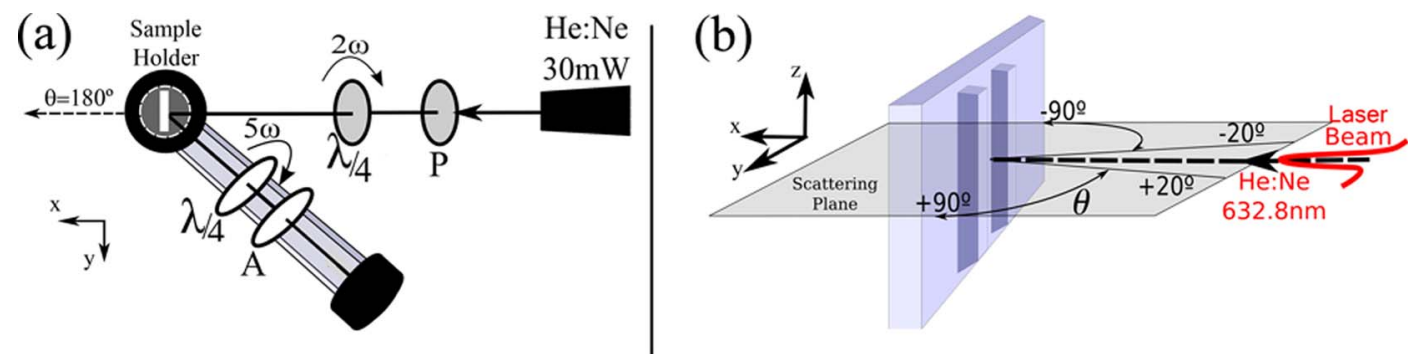

Fig. 1. (Color online) (a) Experimental setup: Positioning (incidence on sample and synchronous waveplates rotations) and measurements are computer controlled. (b) Scattering configuration for a two-rib sample. 


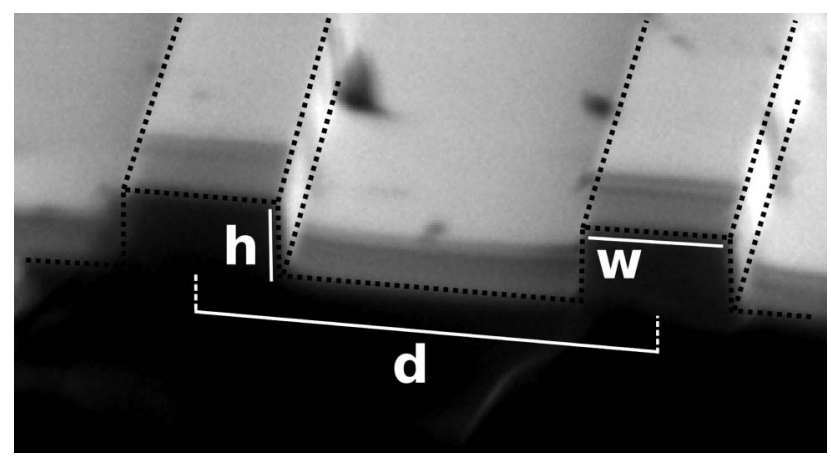

Fig. 2. SEM image of a two-rib structure with dimensions $h=1 \mu \mathrm{m}, w=2 \mu \mathrm{m}$, and $d=6 \mu \mathrm{m}$.

$$
\begin{aligned}
& M_{R}(\phi, \delta, \rho)=M_{R}^{\prime}(\phi, \delta) \\
& \cdot\left(\begin{array}{cccc}
1 & 0 & 0 & 0 \\
0 & \cos (2 \rho) & \sin (2 \rho) & 0 \\
0 & -\sin (2 \rho) & \cos (2 \rho) & 0 \\
0 & 0 & 0 & 1
\end{array}\right) .
\end{aligned}
$$

The retardation parameter, $\delta$, also could be obtained by using a typical polarimetric expression:

$$
\tan (\delta)=\frac{m_{23}-m_{32}}{m_{22}+m_{33}} .
$$

The last operating matrix is $M_{\Delta}$. This matrix informs about the depolarizing processes taking place within the system, mostly produced by multiple scattering or other incoherent processes [15]:

$$
M_{\Delta}\left(d_{i}, a_{i}, z_{i}\right)=\left(\begin{array}{cccc}
1 & 0 & 0 & 0 \\
z_{1} & d_{1} & a_{1} & a_{2} \\
z_{2} & a_{1} & d_{2} & a_{3} \\
z_{3} & a_{2} & a_{3} & d_{3}
\end{array}\right)=\left(\begin{array}{cc}
1 & \mathbf{0}^{T} \\
\mathbf{P}_{\Delta} & m_{\Delta}
\end{array}\right) .
$$

Nine depolarization parameters appear in $M_{\Delta}$, three of them $\left(a_{i}\right)$ closely associated with retardance, another three $\left(z_{i}\right)$ related to diattenuation, and the three diagonal parameters $\left(d_{i}\right)$ referred to as principal depolarization parameters [14], which describe

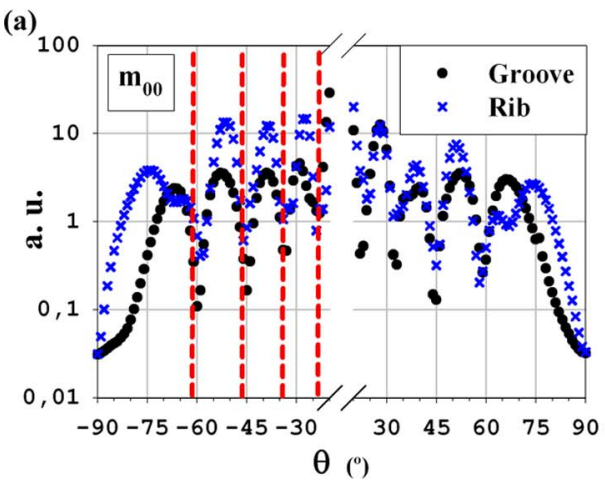

the depolarization capabilities of this polarizer along its principal axes [5]. Note that, in depolarizing systems with $a_{3} \simeq 0$ and $d_{2} \simeq d_{3} \rightarrow 0$, uncertainty in $\delta$ increases [Eq. (4)].

Other matrix operations are very convenient, prior to PD. In order to know if the experimental MMs are pure or show any depolarization traces, it is necessary to apply the purity criterion [14], summarized on Eq. (6). It reveals the kind of matrix under analysis: Eq. (6) is the equality if $M$ is a pure MM and the left side is equal to $m_{00}^{2}$ if $M$ is a completely depolarized MM:

$$
\operatorname{tr}\left(M^{T} M\right) \leq 4 m_{00}^{2} .
$$

Usually, a pure MM has a maximum of seven degrees of freedom, while a depolarizing MM could have up to 16 degrees of freedom. We can use the PD to determine the parameters in a quite straightforward way, approaching the number to the degrees of freedom by solving the dependences of the MM elements. If an experimental matrix is not a pure MM, the $\mathrm{PD}$ produces a new polarimetric description of the system with more than seven independent parameters.

\section{Results}

\section{A. Conventional Light-Scattering Analysis}

Figure $\underline{3}$ represents the evolution of element $m_{00}$ (scattered intensity) for a single and double structures. The inverse scattering problem involves a clear distinction between those structures, and the estimate of the main parameters involved, i.e., $w$, $h$, and $d$. Basic diffraction optics offers a simple comparison with slit and double-slit diffraction patterns (whose minima for $w=3 \mu \mathrm{m}$ and $d=4 \mu \mathrm{m}$ are shown in the plots as a set of dashed lines), and this fit is quite good for the case of grooves. As we can see, the scattering pattern for a single groove corresponds to the pattern for a smaller single rib. In the case of two structures, there are differences only for high scattering angles. This kind of agreement (the slit comparison) is similar to those used in particle-sizing techniques applied to spheres and fibers [16]. However, if parameter $h$ had to be implemented

(b)

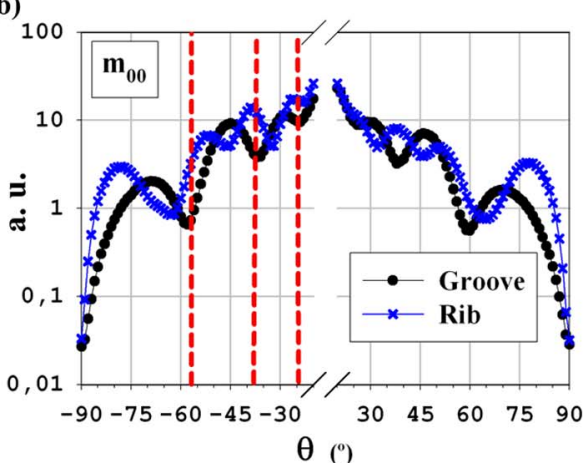

Fig. 3. (Color online) Scattering pattern $\left(m_{00}\right)$ : Comparison with (a) diffraction minima (single structures: $h=1 \mu \mathrm{m}$ and $\left.w=3 \mu \mathrm{m}\right)$ and (b) interference minima (double structures: $h=1 \mu \mathrm{m}, w=3 \mu \mathrm{m}$, and $d=4 \mu \mathrm{m}$ ). 
(to account for height and to distinguish ribs from grooves), the diffraction approach is useless, and we should resort to numerical solutions. In addition, the correct fit of these solutions, for any sign of $h$, is not guaranteed. In the best case, the process is complex, and different for each sample.

\section{B. General MM Analysis}

The MM contains full polarimetric information and must provide other possibilities to be exploited. The angular evolution of the MM elements for two cases of square-profiled single structures is shown in Fig. 4. Both are all-silicon structures with $h=1 \mu \mathrm{m}$, the first with $w=1 \mu \mathrm{m}$ (rib) and the second with $w=3 \mu \mathrm{m}$ (groove). There is a strong matrix symmetry due to the geometry of the scattering system (identical at both sides of the scattering plane) and the properties of MMs for such systems [17], with a polarimetric response close to a block-diagonal plane surface. There is also an angular symmetry around $0^{\circ}$ in each element dependence. This, however, is not as good as expected, showing the presence of inhomogeneities in the fabrication process. But, apart from these considerations, if these matrices contain all information available for our scattering geometry and operating wavelength, its observation and consequent use is not a straightforward issue. For instance, the shape of the patterns corresponding to elements $m_{00}$ and $m_{01}$ (directly related with the linear polarization degree) has been used in the past to size spherical particles or cylindrical fibers [18]. There are, of course, significant differences between these two patterns, corresponding one to a rib, and the other corresponding to a groove, but no systematic difference holds significantly for other rib and groove cases.

\section{PD Analysis}

All the PD parameters corresponding to the two cases shown in Fig. 4 are plotted separately in Fig. 5(a) (rib, $h=1 \mu \mathrm{m}, w=1 \mu \mathrm{m}$ ) and 5(b) (groove, $h=\overline{1 \mu \mathrm{m}}, w=3 \mu \mathrm{m})$. Some preliminary comments in these results include the following.

i. Depolarization effects (third-row parameters in Fig. 5) are especially noticeable where the total scattering intensity, $m_{00}$, has a minimum. These effects appear mainly as a strong departure from 1 in parameters $d_{i}$.

ii. Oscillations in parameter $t$, identical to those of $m_{10}$, is the only important feature in the diattenuation matrix parameters (first row of graph).

iii. Azimuth $(\phi)$ and rotational $(\rho)$ parameters of the retardation matrix (first and third in the second row) are almost null.

In addition to these considerations, if we compare the pure and depolarizing matrices obtained through PD for a single Si rib (see Fig. 6), we can observe that depolarizing effects are not the only cause of differences in the MM of this system with respect to a block-diagonal matrix. It should be noted that results presented in Fig. 5 show that the pure part of the PD performed on the MM of these objects possesses orthogonal eigenpolarizations. One of the most notorious features in these plots is the behavior of $\delta$ (phase retardation between orthogonal components, second parameter in second row). This parameter, related to $m_{i j}(i, j=2,3)$ through Eq. (4), shows a particular angular evolution that is completely different for both structures: each plot contains a dominant slope, which sign is different in each case. For the rib and for all measured ribs, the dominant slope is negative
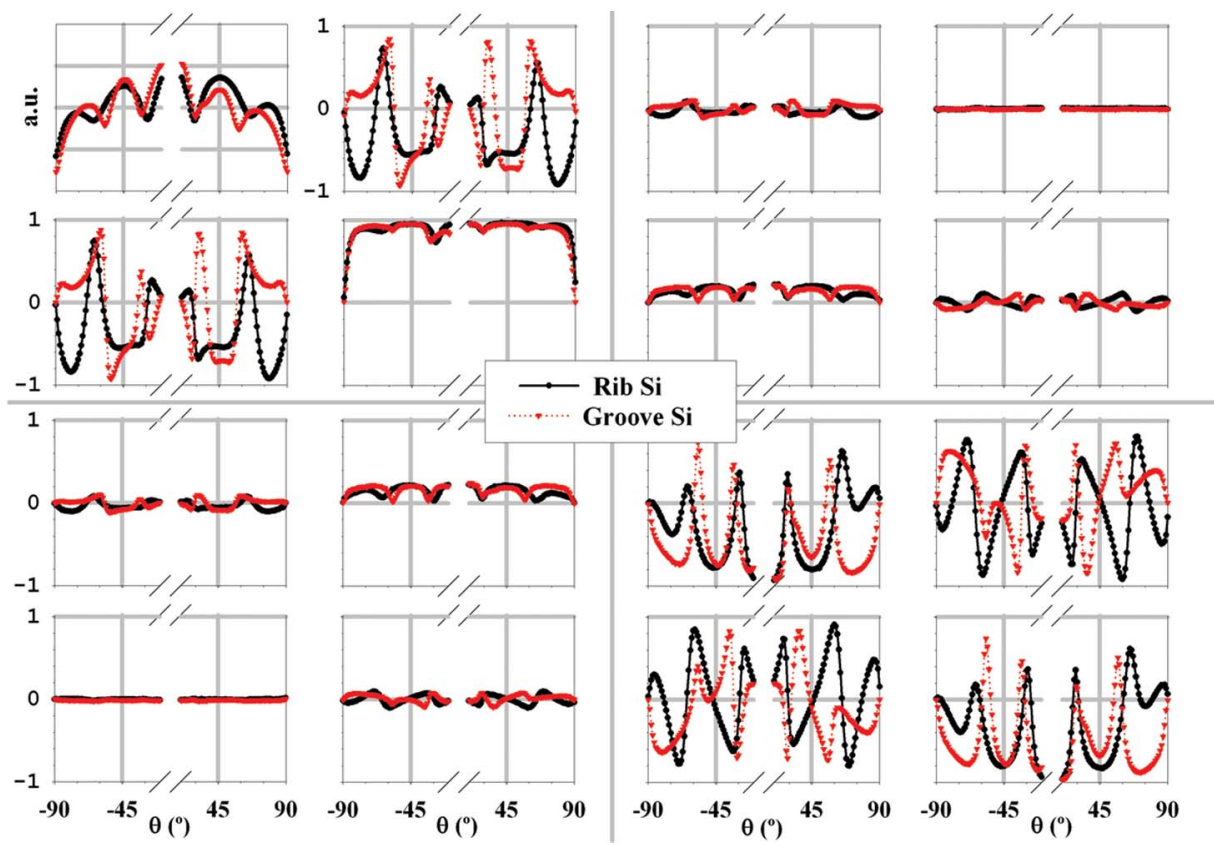

Fig. 4. (Color online) MM elements versus scattering angle $\left(-90^{\circ}\right.$ to $\left.90^{\circ}\right)$ for a single Si rib $(h=1 \mu \mathrm{m}$ and $w=1 \mu \mathrm{m})$ and groove $(h=1 \mu \mathrm{m}$ and $w=3 \mu \mathrm{m}$ ). 
when approaching the specular direction, while for the groove and for all analyzed grooves, the dominant slope is positive. In both cases, the dominant slope is compensated by short angular periods with either a strong slope in the opposite direction or a discontinu- ity. The first derivative of $\delta$ is plotted in Fig. 7 for the cases shown in Figs. 4 and 5, and a black band under the plots indicate the sign of $\delta$ slope. It suggests that the presence of ribs or grooves in a sample can be established by this sole consideration.

\section{(a) $\mathrm{Rib}$}
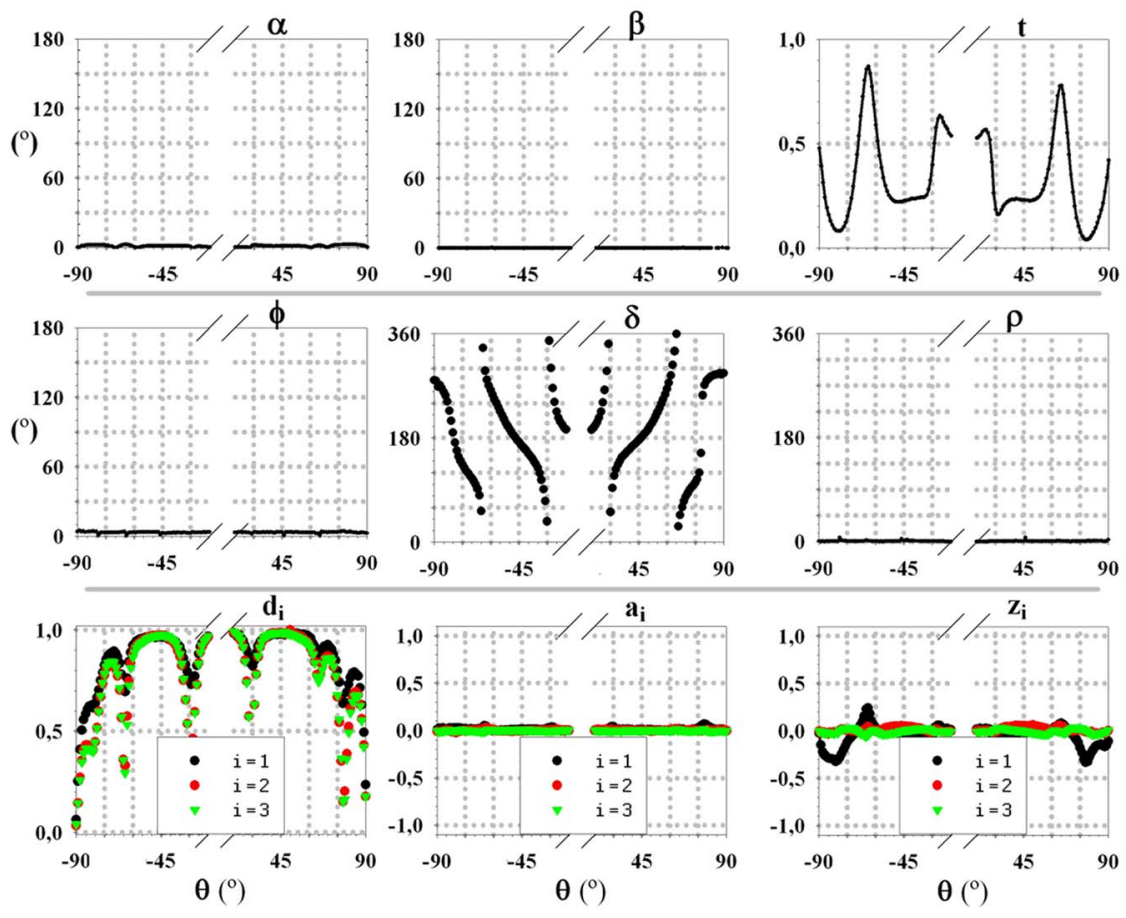

\section{(b) Groove}
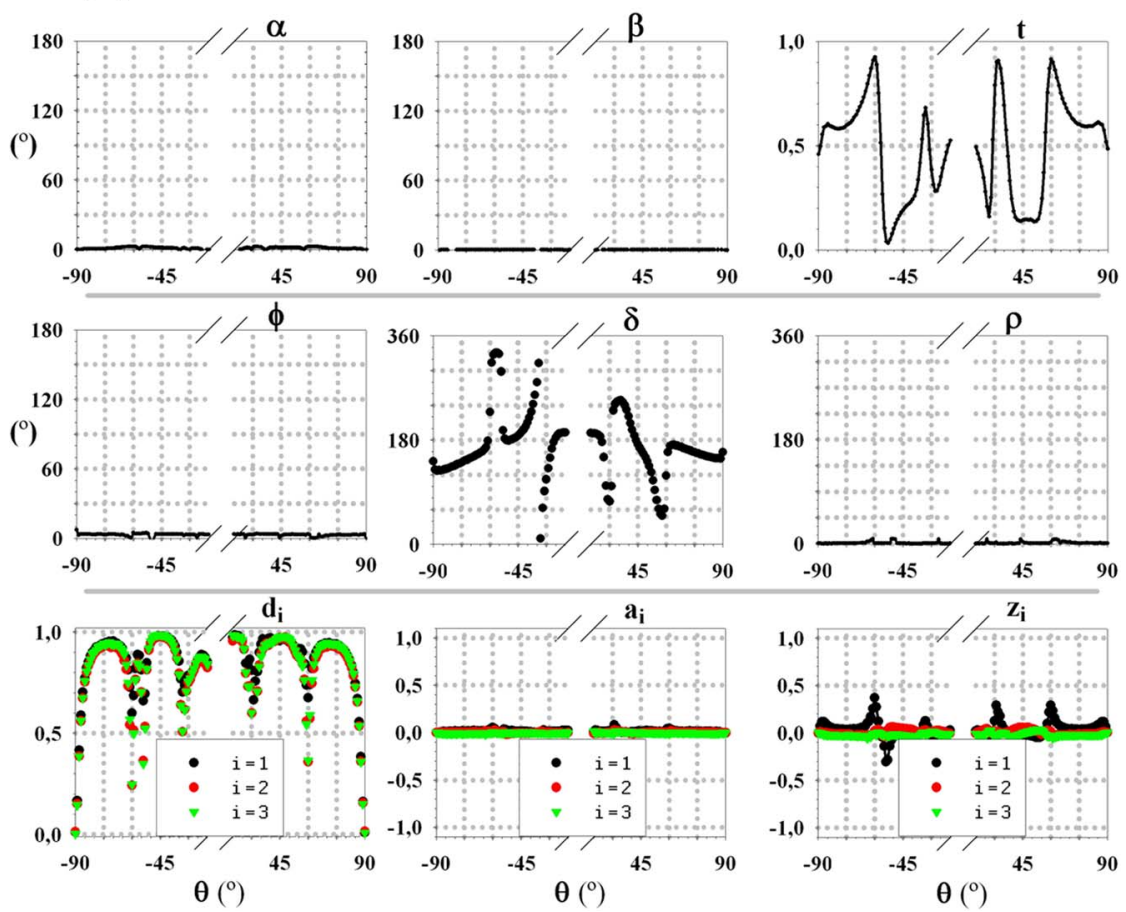

Fig. 5. (Color online) PD parameters for single equivalent structures on Si: (a) rib, $h=1 \mu \mathrm{m}$ and $w=1 \mu \mathrm{m}$; (b) groove, $h=1 \mu \mathrm{m}$ and $w=3 \mu \mathrm{m}$. Top row (diattenuation parameters: $\alpha, \beta$, and $t$ ), center row (retardance parameters: $\varphi, \delta$, and $\rho$ ), bottom row (depolarization parameters: $d_{i}, a_{i}$ and $z_{i}$ ). 


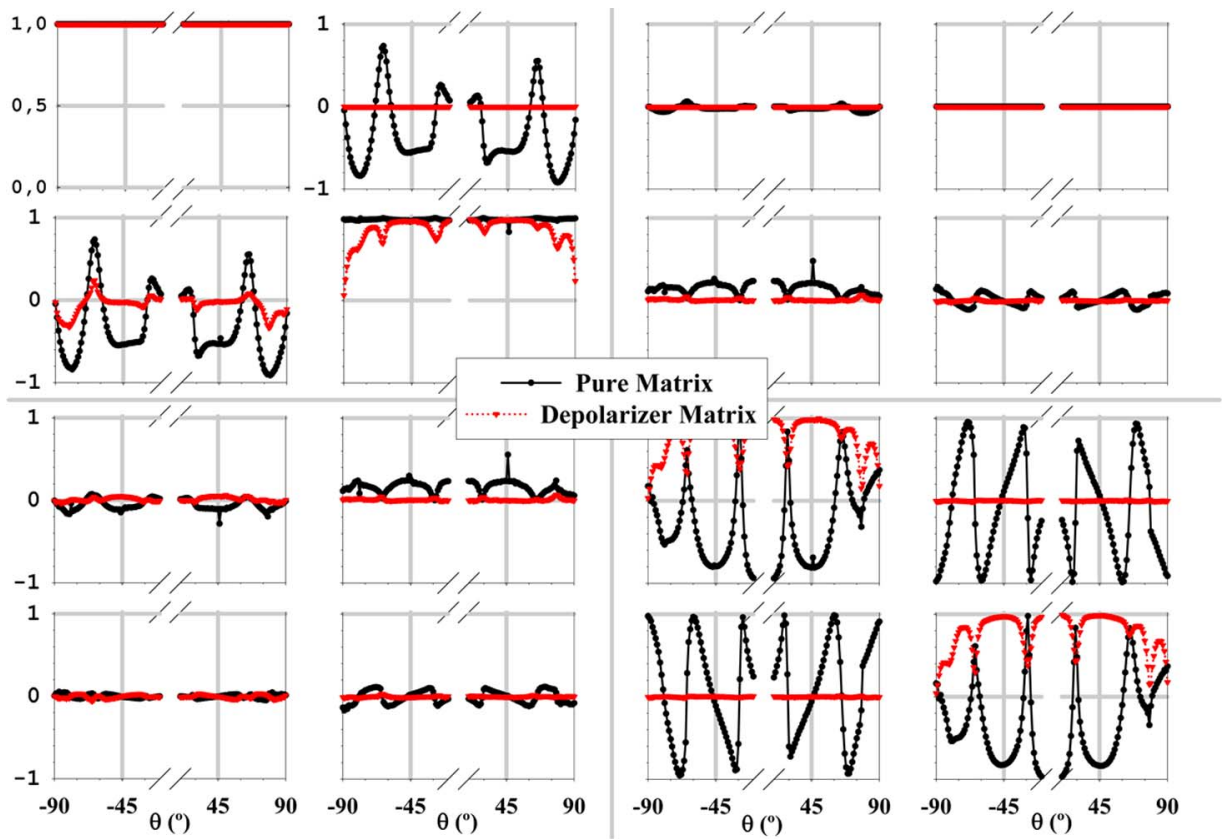

Fig. 6. (Color online) MM elements versus scattering angle $\left(-90^{\circ}\right.$ to $\left.90^{\circ}\right)$ for a single $\mathrm{Si}$ rib $(h=1 \mu \mathrm{m}$ and $w=1 \mu \mathrm{m})$ : pure and depolarizer matrix.

Other examples of the angular evolution of $\delta$, as obtained from PD, together with the sign of its first derivative, are shown in Fig. 8, including Ausputtered and double-structured cases (dimensions are specified in the caption).

\section{Rule of Thumb}

In order to evaluate this interesting property, we calculate, for each measured scattering region (for normal incidence, and in $1^{\circ}$ steps), the difference between the number of measurements with positive and negative local derivative. A new parameter, $\Upsilon_{R-G}$, is defined as

$$
\Upsilon_{R-G}=\frac{\sum_{\theta>0} \frac{\Delta \delta_{i}}{\left|\Delta \delta_{i}\right|}-\sum_{\theta<0} \frac{\Delta \delta_{i}}{\left|\Delta \delta_{i}\right|}}{N},
$$

where $N$ is the total number of steps, and the first $(\theta>0)$ and second $(\theta<0)$ summatory corresponds respectively to the right and left scattering regions.
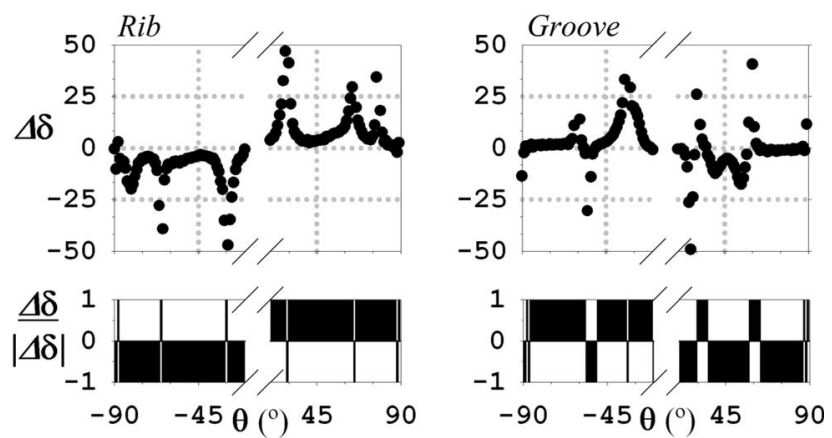

Fig. 7. $\delta$ slope for a single rib/groove obtained through PD. The dominant sign of the slope is shown in black bars (increment $\Delta \delta$ corresponds to $\Delta \theta=1^{\circ}$ ).
Defined in this way, $\Upsilon_{R-G} \in[-1,1]$ and a positive overall would indicate the presence of ribs, while a negative value would be associated to the presence of grooves. Values approaching zero would indicate uncertainty. Table 1 has been constructed from all available measurements corresponding to the set of structures made on our wafer. The only case
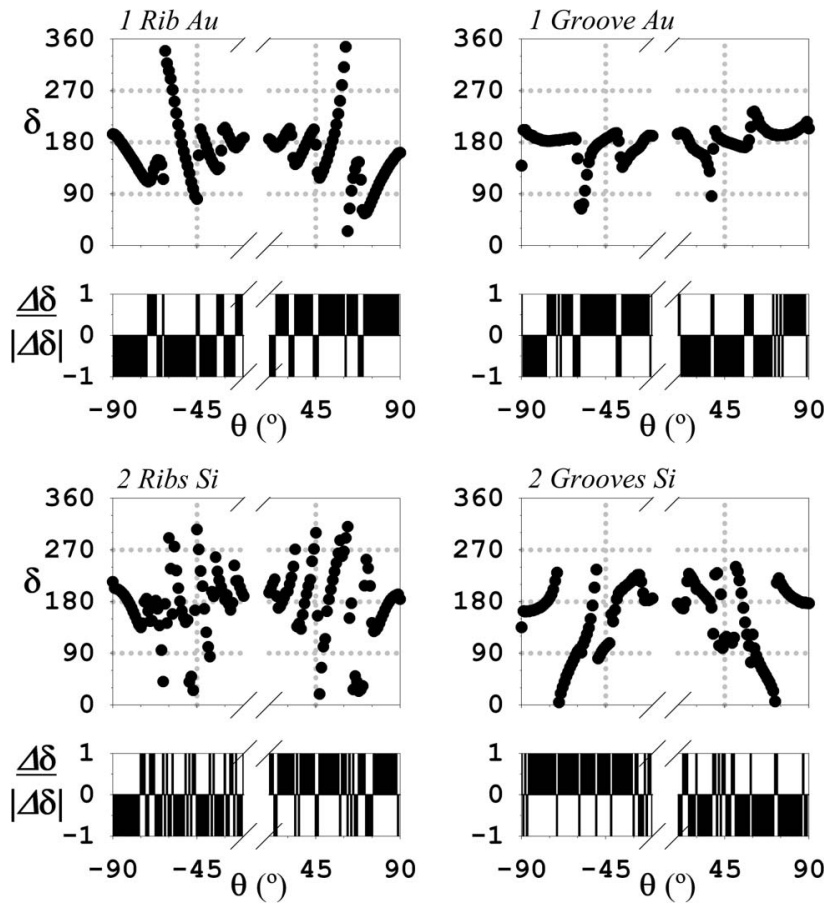

Fig. 8. Values of $\delta$ and its slope sign for different geometries and materials. Upper cases, Au-sputtered single rib (left) and groove (right) of $h=1 \mu \mathrm{m}$ and $w=3 \mu \mathrm{m}$. Bottom cases, two parallel Si ribs/grooves of $h=1 \mu \mathrm{m}, w=3 \mu \mathrm{m}$, and $d=7 \mu \mathrm{m}$. 
Table 1. Values of $\Upsilon_{R-G}$ for 2D Square-Profiled Structures $h=1 \mu \mathrm{m}^{a}$

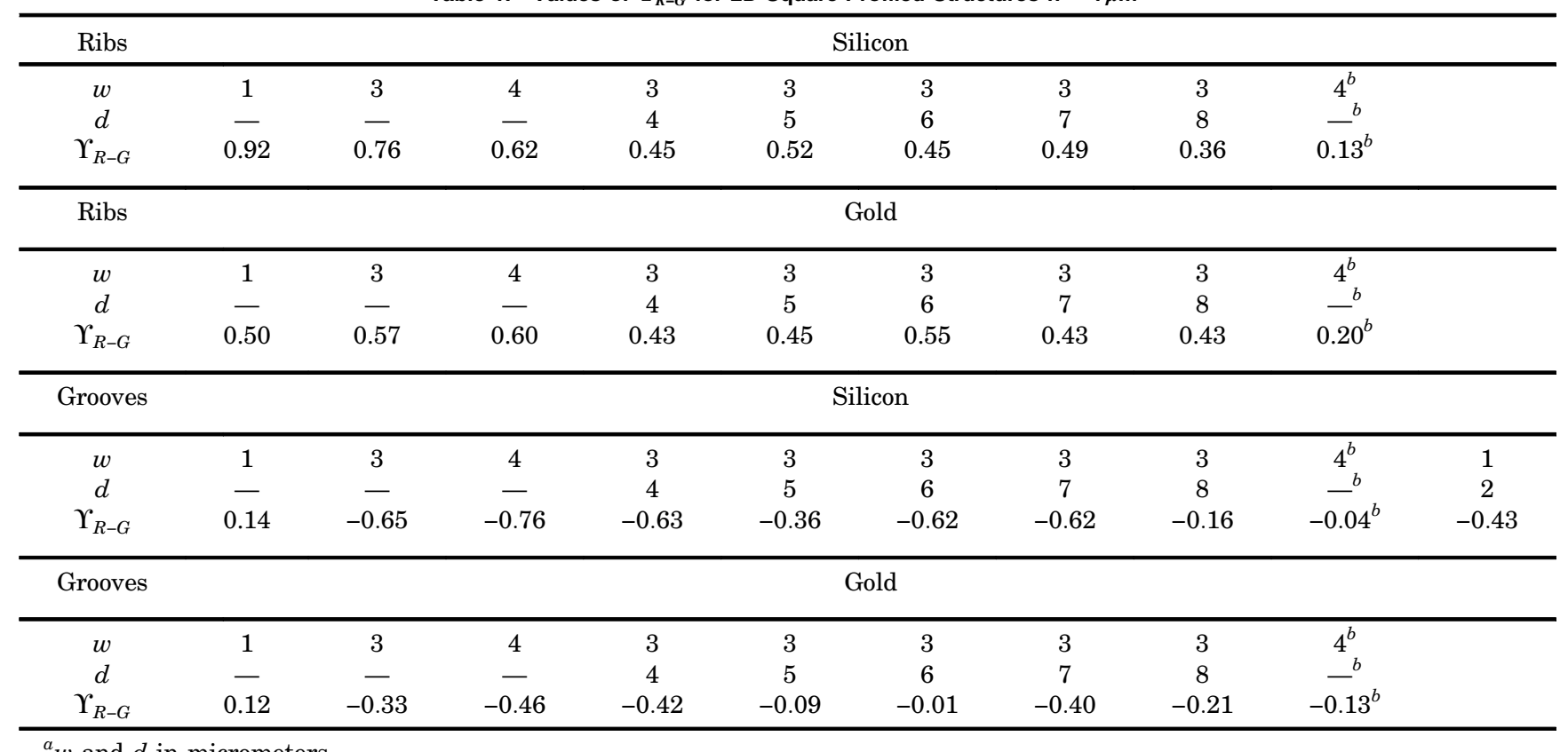

${ }^{a} w$ and $d$ in micrometers.

${ }^{b} h=2 \mu \mathrm{m}$.

( $h=1 \mu \mathrm{m}$ and $w=1 \mu \mathrm{m}$, groove) that does not fulfill the sign criterion for $\Upsilon_{R-G}$ corresponds to the sample for which the fabrication defects are more severe, producing a shape with a strong departure from the square profile. The importance of this result lies in the fact that all samples follow the same rule independently of the width, number of elements, distance between them, height/depth, or material ( $\mathrm{Si}$ or Au sputtered). In most cases $(>85 \%), \Upsilon_{R-G}$ produced correct predictions with $\left|\Upsilon_{R-G}\right| \geq 0.13$ and in two thirds of cases with $\left|\Upsilon_{R-G}\right| \geq 0.40$.

If we consider the possibility of applying this rule to a single MM element connected to $\delta$, like $m_{23}$, in all cases analyzed $m_{23}$ indicates uncertainty when the $\delta$ slope analysis show conclusive values. Obviously the rule of- thumb reached by handling PD parameter $\delta$ can also be obtained by using elements $m_{i j}[i, j=2,3$, see Eq. (4)], as will happen to any condition established on PD parameters. The advantage of PD lies in its potential for obtaining relevant conditions because of the physical meaning of each PD parameter; in other words, the rule of thumb was found after the PD process.

\section{Summary and Conclusions}

In this research, we used PD to interpret information from MMs. Using a DRCP polarimeter, these matrices were experimentally obtained for several flat surface systems containing square-profiled structures that are either built on (ribs) or dug in (grooves) the surface. As an application of PD, we have established a polarimetric criterion that allows us to distinguish between both types of surface microdefects (ribs or grooves) in a systematic way, regardless of size or composition of the sample.
Consequently, PD applied to experimental MM has proved to be a useful tool for analyzing and processing the polarimetric information by isolating the effects produced by variations in a particular feature of the system. Specifically, direct application to surface defect characterization by analyzing the angular derivative of the retardation, $\delta$, produces conclusive estimates of shape for a square-profiled $2 \mathrm{D}$ surface structure. Furthermore, as PD is an algebraic tool, the rule is applicable to conventional polarimetry using the correct estimation of $\delta$ [Eq. (4)]. This result is an excellent example of the potential of PD, because it is able not only to extract the depolarization but also to analyze the remaining (pure) matrix.

This research has been supported by the Ministry of Education of Spain under project FIS2010-21984. Photolithographic samples were provided by TEKNIKER (Eibar, Spain).

\section{References}

1. G. Videen and W. S. Bickel, "Light-scattering mueller matrix for a rough fiber," Appl. Opt. 31, 3488-3492 (1992).

2. S. N. Savenkov, L. T. Mishchenko, R. S. Muttiah, Y. A. Oberemok, and I. A. Mishchenko, "Mueller polarimetry of virus-infected and healthy wheat under field and microgravity conditions," J. Quant. Spectrosc. Radiat. Transfer 88, 327-343 (2004).

3. Y. Cui and R. M. A. Azzam, "Applications of the normalincidence rotating-sample ellipsometer to high- and lowspatial-frequency gratings,” Appl. Opt. 35, 2235-2238 (1996).

4. J. J. Gil and E. Bernabeu, "Obtainment of the polarizing and retardation parameters of a non-depolarizing optical system from the polar decomposition of its mueller matrix," Optik 76, 67-71 (1987).

5. S. Y. Lu and R. A. Chipman, "Interpretation of mueller matrices based on polar decomposition," J. Opt. Soc. Am. A 13, 1106-1113 (1996). 
6. J. Chung, W. Jung, M. J. Hammer-Wilson, P. Wilder-Smith, and $\mathrm{Z}$. Chen, "Use of polar decomposition for the diagnosis of oral precancer," Appl. Opt. 46, 3038-3045 (2007).

7. M. K. Swami, S. Manhas, P. Buddhiwant, N. Ghosh, A. Uppal, and P. K. Gupta, "Polar decomposition of $3 \times 3$ mueller matrix: a tool for quantitative tissue polarimetry," Opt. Express 14, 9324-9337 (2006).

8. C. Collet, J. Zallat, and Y. Takakura, "Clustering of mueller matrix images for skeletonized structure detection," Opt. Express 12, 1271-1280 (2004).

9. M. Foldyna, A. De Martino, R. Ossikovski, E. Garcia-Caurel, and C. Licitra, "Characterization of grating structures by Mueller polarimetry in presence of strong depolarization due to finite spot size," Opt. Commun. 282, 735-741 (2009).

10. M. H. Smith, "Optimization of a dual-rotating-retarder Mueller matrix polarimeter," Appl. Opt. 41, 2488-2493 (2002).

11. R. M. A. Azzam, "Photopolarimetric measurement of the Mueller matrix by Fourier analysis of a single detected signal," Opt. Lett. 2, 148-150 (1978).
12. S. R. Cloude, "Group theory and polarization algebra," Optik 75, 26-36 (1986).

13. J. M. Sanz, P. Albella, F. Moreno, J. M. Saiz, and F. González, "Application of the polar decomposition to light scattering particle systems," J. Quant. Spectrosc. Radiat. Transfer 110, 1369-1374 (2009).

14. J. J. Gil, "Polarimetric characterization of light and media," Eur. Phys. J. Appl. Phys. 40, 1-47 (2007).

15. M. Foldyna, E. García-Caurel, R. Ossikovski, A. D. Martino, and J. Gil, "Retrieval of a non-depolarizing component of experimentally determined depolarizing mueller matrices," Opt. Express 17, 12794-12806 (2009).

16. J. de la Peña, J. Saiz, P. Valle, F. González, and F. Moreno, "Tracking scattering minima to size metallic particles on flat substrates," Part. Part. Syst. Charact. 16, 113-118 (1999).

17. H. Hulst, Light Scattering by Small Particles (Dover, 1981).

18. J. L. de la Peña, F. González, J. M. Saiz, F. Moreno, and P. J. Valle, "Sizing particles on substrates. a general method for oblique incidence," J. Appl. Phys. 85, 432-438 (1999). 